\title{
Columella Lengthening with a Full-Thickness Skin Graft for Secondary Bilateral Cleft Lip and Nose Repair
}

\author{
Yoon Seok Lee ${ }^{1}$, Dong Hyeok Shin ${ }^{1}$, Hyun Gon Choi $^{1}$, Jee Nam Kim ${ }^{1}$, Myung Chul Lee ${ }^{1}$, \\ Soon Heum Kim², Cheol Keun Kim², Dong In Jo², Ki Il Uhm ${ }^{1}$ \\ ${ }^{1}$ Department of Plastic and Reconstructive Surgery, Konkuk University Medical Center, Konkuk University School of Medicine, Seoul; \\ ${ }^{2}$ Department of Plastic and Reconstructive Surgery, Konkuk University Chungju Hospital, Konkuk University School of Medicine, Chungju, \\ Korea
}

Background Various techniques for lengthening short columellae have been used for bilateral cleft nose repair. However, previous methods have not yielded satisfactory results. We performed a full-thickness skin graft to lengthen short columellae during secondary cleft nose repair in adult patients.

Methods Ten bilateral cleft lip and nose patients underwent secondary cheiloplasty with open rhinoplasty between July 2008 and August 2014. The patients underwent a fullthickness skin graft on the medial crura to elongate the columella. The average age of the patients at the time of surgery was 22.2 years. Nasal profiles were evaluated before and after the operation using the photogrammetric method.

Results The nasal profiles were improved in all patients, and all skin grafts were well taken, with the exception of one patient. Columellar height, nostril height, and columella-lip angle increased, and nasal width decreased significantly. The ratios of columellar height to nasal height, columellar height to nasal width, and nasal height to nasal width increased to a statistically significant extent.

Conclusions Columella lengthening with a full-thickness skin graft is a simple and effective method for the repair of severely short columellae in bilateral cleft nose patients. We had satisfactory outcomes, with good color matching and aesthetically pleasing contours.

Keywords Cleft lip / Nose / Skin transplantation

\author{
Correspondence: Ki II Uhm \\ Department of Plastic and \\ Reconstructive Surgery, Konkuk \\ University Medical Center, Konkuk \\ University School of Medicine, 120-1 \\ Neungdong-ro, Gwangjin-gu, Seoul \\ 05030, Korea \\ Tel: +82-2-2030-5235 \\ Fax: +82-2-2030-5249 \\ E-mail: distro123@gmail.com
}

No potential conflict of interest relevant to this article was reported.

Received: 1 Jun 2015 • Revised: 27 Aug 2015 • Accepted: 1 Sep 2015

pISSN: 2234-6163 • elSSN: 2234-6171 • http://dx.doi.org/10.5999/aps.2015.42.6.704 • Arch Plast Surg 2015;42:704-708

\section{INTRODUCTION}

Bilateral cleft nose deformities involve a short columella, wide nostrils, flared alar rims, and a poorly projected nasal tip. The short columella plays a major role in the overall bilateral cleft nose deformity and should be lengthened. Various techniques for lengthening short columellae have been developed, including V-Y advancement flaps, reverse V-Y advancements connected to bilateral bipedicle flaps, forked flaps, and composite grafts. However, these methods have a limited scope of application, create an abnormal columellar shape and scarring in the columella, and are difficult to perform [1-4]. 
In this study, a full thickness skin graft from the postauricular area was used to lengthen the short columella during secondary cleft nose repair in adult patients. This technique can be easily performed and used in patients with a severely short columella. Furthermore, we anticipated that columella lengthening would provide several benefits in addition to elongation of the columellae and increased nostril height.

\section{METHODS}

A retrospective review was performed of 10 bilateral cleft lip and nose patients who underwent secondary cheiloplasty with open rhinoplasty between July 2008 and August 2014. The patients underwent a full-thickness skin graft to elongate the columella. All operations were performed by a single plastic surgeon (K.I.U.). Five male patients and five female patients were included, with an average age at the time of surgery of 22.2 years (range, 17-33 years). The average duration of follow-up was 14.3 months (Table 1).

Photographs were taken preoperatively and three months postoperatively for all patients. Using these photographs, we measured the pronasale, columellar peak, subnasale, and alar curvature point [5]. The columellar height, nasal height, nostril height,

\section{Fig. 1. Nasal profile}

$A 1$, right nostril height; $A 2$, left nostril height; $B 1$, right nostril width; $\mathrm{B} 2$, left nostril width; $\mathrm{C}$, columellar width; $\mathrm{CL}$, columella-lip angle.
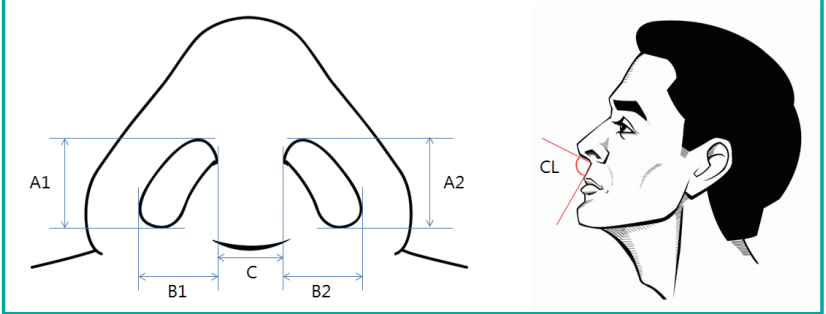

columellar width, nasal width, and nostril width were measured from worm's eye view photographs. The columella-lip angle was measured using lateral-view photographs (Fig. 1) [6].

The means and standard deviations of the 12 variables were calculated before and after the operation. The Wilcoxon ranksum test was performed, and P-values $<0.05$ were considered to indicate statistical significance.

\section{Surgical technique}

All operations were performed under general anesthesia. After secondary cheiloplasty was performed for upper lip and philtrum deformities, open rhinoplasty was performed. Marginal and transcolumellar incisions were made on the nasal mucosa and midcolumella using a No. 15 blade to elevate the cutaneous flap. Dissection was performed to the supraperichondrial plane with Metzenbaum scissors and was continued extensively below the perichondrium to expose the septal cartilage. The septal cartilage for the septal extension graft was harvested via a caudal

\section{Fig. 2. Intraoperative photograph}

A 19-year-old female with a bilateral cleft lip and nose deformity (patient 6) underwent secondary cheiloplasty and open rhinoplasty. After the septal extension graft, both medial crura were sutured to prepare a flat bed. A full-thickness skin graft with a tie-over dressing was performed on the vascularized tissue of the medial crura.

(A) Frontal view. (B) Right lateral view.

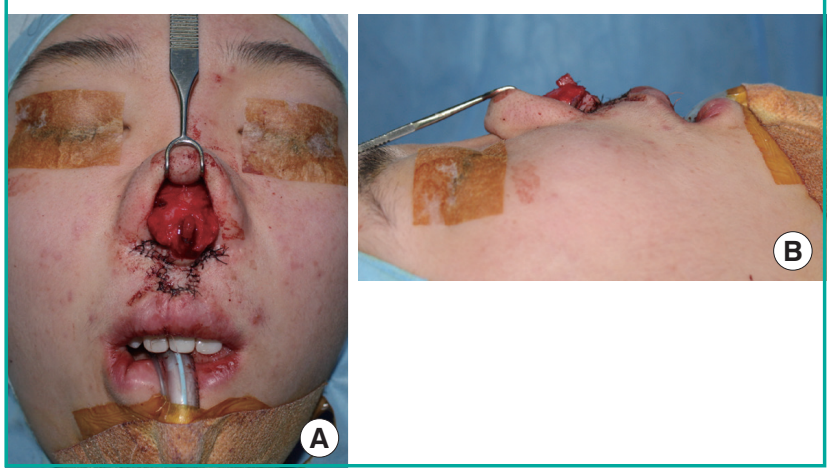

Table 1. Demographic and clinical information of the patients included in this study

\begin{tabular}{|c|c|c|c|c|c|c|c|c|}
\hline Patient & Sex & Age (yr) & Cleft type & $\begin{array}{c}\text { Septal extension } \\
\text { graft }\end{array}$ & Tip plasty & $\begin{array}{c}\text { Dorsal } \\
\text { augmentation }\end{array}$ & Complication & $\begin{array}{c}\text { Follow-up } \\
\text { duration (mo) }\end{array}$ \\
\hline 1 & Male & 19 & Complete and complete & Septal cartilage & Conchal cartilage & Dermis & - & 3 \\
\hline 2 & Female & 23 & Complete and complete & Costal cartilage & Dermis & Dermis & - & 6 \\
\hline 3 & Male & 25 & Complete and complete & Septal cartilage & Dermis & Dermis & - & 3 \\
\hline 4 & Female & 29 & Complete and complete & Septal cartilage & Dermis & None & - & 32 \\
\hline 5 & Male & 21 & Complete and complete & Septal cartilage & Conchal cartilage & Silicone & - & 7 \\
\hline 6 & Female & 19 & Complete and complete & Septal cartilage & Dermis & Dermis & - & 3 \\
\hline 7 & Male & 17 & Complete and complete & Septal cartilage & Conchal cartilage & None & - & 48 \\
\hline 8 & Female & 18 & Complete and complete & Septal cartilage & Conchal cartilage & None & Secondary healing scar & 8 \\
\hline 9 & Male & 33 & Complete and complete & Costal cartilage & Costal cartilage & Dermis & Graft necrosis & 3 \\
\hline 10 & Female & 18 & Complete and complete & Septal cartilage & Conchal cartilage & Dermis & - & 30 \\
\hline
\end{tabular}


septal approach, leaving the dorsal and caudal sides of the cartilage intact. The harvested septal cartilage was sutured to the anterior septum with 5-0 Prolene. If the patient did not have enough septal cartilage, costal cartilage was used for the septal extension graft (Fig. 2). Previously harvested dermis or silicone was sutured onto the nasal dorsum using a pull-out onlay graft for dorsal augmentation. Tip plasty was performed with the previous harvested dermis or conchal cartilage. After open rhinoplasty, as all patients showed a severely short columella and suturing the columellar flap directly could lead to unsatisfying tip projection, we measured the extent of the columellar gaps and harvested full-depth skin from the postauricular area. After preparing a flat recipient bed by suturing both sides of the medial crura, a full-thickness skin graft with a tie-over dressing was performed.

\section{RESULTS}

The nasal profiles were improved in all patients. The columellar and nostril heights were increased. Most patients showed improved tip projection (Fig. 3). All skin grafts were well taken with minimal contraction, with the exception of one patient who experienced total necrosis. Another patient showed a secondary healing scar on the grafted columella. No patient showed a noticeable trap-door effect.

Several changes in the nasal profile were statistically significant. Preoperative and postoperative mean nasal profile measurements are presented in Table 2. Columellar height, nostril height, and the columella-lip angle increased, and the nasal width decreased. The ratios of columellar height to nasal height, columellar height to nasal width, and nasal height to nasal width increased significantly.

\section{DISCUSSION}

In bilateral cleft lip and nose patients, the presence of a short columella has led surgeons to perform various surgical techniques in order to correct this deformity. McIndoe and Rees [1]

\section{Fig. 3. Photographs of patient 5}

The patient underwent secondary cheiloplasty and open rhinoplasty with a full-thickness skin graft to elongate the columella. (A, B) Preoperative views. (C, D) Six-month postoperative views.
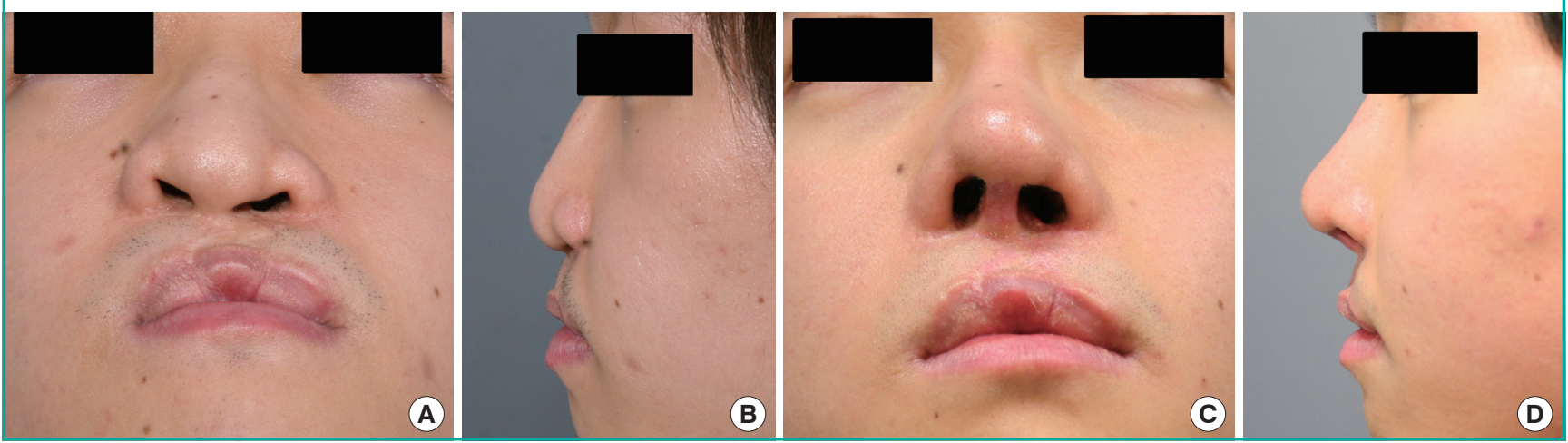

Table 2. Nasal profile changes after surgery

\begin{tabular}{|c|c|c|c|c|}
\hline Nasal profile & Preoperative & Postoperative & Change & P-value \\
\hline Columellar height (mm) & $6.95 \pm 1.69$ & $11.37 \pm 0.81$ & $4.42 \pm 1.86$ & 0.002 \\
\hline Columellar width (mm) & $9.21 \pm 1.28$ & $8.70 \pm 1.75$ & $-0.51 \pm 1.50$ & 0.311 \\
\hline Nostril height, right (mm) & $6.70 \pm 1.47$ & $9.66 \pm 1.07$ & $2.96 \pm 1.53$ & 0.002 \\
\hline Nostril height, left (mm) & $6.87 \pm 1.83$ & $9.86 \pm 1.59$ & $2.99 \pm 1.19$ & 0.002 \\
\hline Nostril width, right (mm) & $10.36 \pm 2.32$ & $9.35 \pm 1.99$ & $-1.01 \pm 1.30$ & 0.055 \\
\hline Nostril width, left (mm) & $10.59 \pm 1.91$ & $9.46 \pm 1.42$ & $-1.13 \pm 1.12$ & 0.008 \\
\hline Nasal height (mm) & $24.27 \pm 2.39$ & $26.36 \pm 2.63$ & $2.09 \pm 3.34$ & 0.131 \\
\hline Nasal width (mm) & $46.09 \pm 5.14$ & $43.00 \pm 4.76$ & $-3.09 \pm 1.65$ & 0.002 \\
\hline Columella-lip angle $\left({ }^{\circ}\right)$ & $99.24 \pm 10.02$ & $108.24 \pm 10.48$ & $9.00 \pm 3.91$ & 0.002 \\
\hline Columellar height/nasal height & $0.29 \pm 0.06$ & $0.44 \pm 0.06$ & $0.15 \pm 0.07$ & 0.002 \\
\hline Columellar height/nasal width & $0.15 \pm 0.05$ & $0.27 \pm 0.04$ & $0.11 \pm 0.05$ & 0.002 \\
\hline Nasal height/nasal width & $0.53 \pm 0.07$ & $0.62 \pm 0.04$ & $0.08 \pm 0.08$ & 0.010 \\
\hline
\end{tabular}


described a technique for lengthening an abnormally short columella using V-Y advancement flaps of the prolabial scars. Cronin [2] reported reverse V-Y advancement connected to bilateral bipedicle flaps for columellar lengthening. Millard [3] described the use of forked flaps, banked at the time of the lip repair, to correct the columellar deficiency. However, these techniques involve using nearby tissue to lengthen a short columella and cannot provide sufficient tissue for patients with a severely short columella. Furthermore, these methods result in an unsightly scar or an abnormally wide columella. Cheon and Park [4] reported columella elongation using a composite graft instead of a local skin flap. They had satisfying results, especially for patients with a severely short columella, but reported the need to develop more refined surgical techniques.

In patients with a severely shortened columella, elongation of the columella is necessary to achieve the desired shape of the nose without any tension or distortion. Local flap coverage has been found to have limitations when performed in such cases. In the current study, we performed open rhinoplasty to correct secondary cleft nose deformities as well as a full-thickness skin graft to lengthen the short columella. The bed of skin graft was vascularized tissue on the perichondrium of the medial crura. Donor tissue was harvested from the postauricular area without any fat underneath the dermis.

Compared to composite grafts, our technique was a simple and effective method for elongating severely short columellae. The full-thickness skin graft donor tissue was easier to harvest than a composite graft and had minimal donor site morbidity. The donor site scar was invisible on the frontal view. Moreover, the tissue of the full-thickness skin graft is more vascularized and less vulnerable to ischemic conditions than the tissue used in composite grafts.

Although skin grafts could produce several problems related to color mismatch, depression, stepping, and unpredictable results, most patients in our study showed satisfactory color matching and a good contour without stepping (Fig. 4), except for one patient who had a secondary healing scar at the graft site (Fig.

\section{Fig. 4. Photographs of patient 1}

The patient underwent secondary cheiloplasty and open rhinoplasty with a full-thickness skin graft to elongate the columella. The grafted skin shows satisfactory color matching and good contour. (A) Preoperative view. (B) Three-month postoperative view.
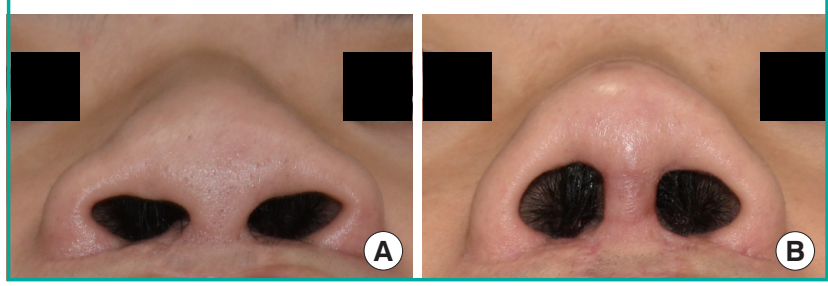

5A) and one patient who had total necrosis that was cured by secondary intention with daily dressing (Fig. 5B). We hypothesize that a well-vascularized flat wound bed and support from the septal extension graft led to good results. After the septal extension graft, suturing both sides of the medial crura resulted in a flat wound bed. Moreover, the presence of well-vascularized tissue on the perichondrium helped the graft take. Cheon and Park [4] reported suturing the cartilaginous portion of the composite tissue to the septum in order to support the columella. In this study, the septal or costal cartilage for the septal extension graft supported the columella instead of the conchal cartilage, which is used in composite grafts.

Through columella lengthening with a full-thickness skin graft, we modified not only the columella and nostril height, which is directly related to columella lengthening, but also nasal width, the columella-lip angle, and the ratio of columellar height to nasal height. In an ideal nasal profile, the columellar height should constitute approximately two thirds of the nasal tip projection [7]. The postoperative ratio of columellar height to nasal height was $0.44 \pm 0.06$, which was greater than preoperative ratio $(0.29 \pm 0.06)$. Although the profiles of patients did not come close to the ideal nasal profile, the operations did lead to meaningful improvement in this regard.

Previous studies have reported that columella lengthening procedures led to a significant decrease in the nasolabial angle $[4,6]$. However, our results were the opposite, with a mean columella-lip angle of $99.24 \pm 10.02$ preoperatively and 108.24 \pm 10.48 postoperatively. This discrepancy may have occurred because our study involved significantly older patients than were included in previous studies (21.75 years vs. 7.3 and 6.5 years, respectively). We suggest that as patients with bilateral cleft lip and nose become older, their obtuse columella-lip angles become acute, such that an open rhinoplasty with a septal extension graft and tip plasty can provide a greater columella-lip angle.

\section{Fig. 5. Complications}

(A) An 18-year-old female with a bilateral cleft lip and nose deformity (patient 7) underwent secondary cheiloplasty and open rhinoplasty with a full-thickness skin graft to elongate the columella. A postoperative view at eight-months shows a secondary healing scar on the grafted tissue. (B) A 33-year-old male with a bilateral cleft lip and nose deformity (patient 8) underwent the same operation. A postoperative view at 15 days shows total necrosis of the graft.
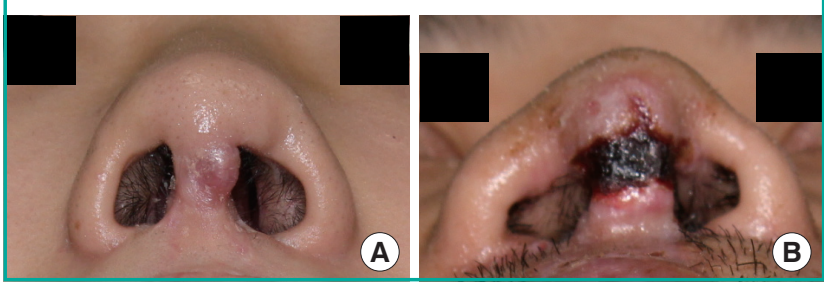
Some patients are reluctant to risk scar formation around the columella and/or do not have sufficient conchal cartilage for a composite graft. Moreover, patients with a severely shortened columella might not have enough nearby tissue for a local flap to be performed. In such cases, columella lengthening with a full-thickness skin graft should be considered.

In order to improve the overall success rate in terms of grafts that are taken, careful dissection is needed, especially when dissecting the supraperichondrial plane around the medial crura. The vascularized tissue on the medial crura must be conserved. Compressive dressing to immobilize the graft and an accurate wound margin approximation to avoid depression or stepping are also necessary.

This study had several limitations. First, the average follow-up period was 14.3 months, which is a relatively short time. Some patients underwent only three months of follow-up, which was not enough time to evaluate the scar maturation. Second, the number of patients recruited was small and further research is necessary.

Columella lengthening with a full-thickness skin graft is a simple and effective method, with minimal donor site morbidity, for the repair of secondary bilateral cleft nose deformities in patients with a severely short columella. We demonstrated that columella lengthening with a full-thickness skin graft during secondary cleft nose correction in bilateral cleft lip patients produced excellent outcomes in terms of columellar height, nostril height, nasal width, the columella-lip angle, and the ratio of columellar height to nasal height.

\section{REFERENCES}

1. McIndoe SA, Rees TD. Synchronous repair of secondary deformities in cleft lip and nose. Plast Reconstr Surg Transplant Bull 1959;24:150-62.

2. Cronin TD. Lengthening columella by use of skin from nasal floor and alae. Plast Reconstr Surg Transplant Bull 1958; 21:417-26.

3. Millard DR. Bilateral cleft lip and a primary forked flap: a preliminary report. Plast Reconstr Surg 1967;39:59-65.

4. Cheon YW, Park BY. Long-term evaluation of elongating columella using conchal composite graft in bilateral secondary cleft lip and nose deformity. Plast Reconstr Surg 2010; 126:543-53.

5. Lindsay WK, Farkas LG. The use of anthropometry in assessing the cleft-lip nose. Plast Reconstr Surg 1972;49:28693.

6. Masuoka H, Kawai K, Morimoto N, et al. Conchal cartilage graft for correction of bilateral cleft lip nasal deformities during childhood. Plast Reconstr Surg Glob Open 2014;2:e104.

7. Sheen JH, Sheen AP. Aesthetic rhinoplasty. St. Louis: Mosby; 1987. 